gical point of view.

1) cranium bifidum cysticum and spina bifida cystica.

These malformations are subdivided into meningocele and encephaloor meningocele. Cranium bifidum cysticum should be resected and repaired in early postnatal stage. Skull defect might be remained as long as life, but cranioplasty is not required. Prognosis of cranium bifidum cystica is better than the spina bifida cystica.

Spina bifida cystica is also resected within 6-12 months of age, and complete repair of the dura and fascial layer is the most important. Spina bifida cystica, especially in the lower spines is apt to get severely infected or to result in paresis of lower extremities and/or sphincter disturbances. In such a severe instance, functional prognosis is very poor even through operation is successfully performed.

It should be emphasized that ventriculo-venous shunting should be performaed in these malformations as soon as hydrocephalus develops.

2) congenital defect of the corpus callosum.

Pneumoencephalographic and angiographic findings are presented.

3) Arnold-Chiari malformation.

It is important in treatment of this malformation that dura mater should be closed or dural plasty should be performed with fascia after extensive suboccipital decompression including laminectomy of the first and second cervical vertebrae.

4) Dandy-Walker's synd rome.

Ventriculo-venous shunting operations should be performed as in hydrocephalus. It is not recommended to resect widely the cyst of the fourth ventricle. From a clinical therapeutic stand of view, it is proposed that postnatal occlusion of the apertures of the forth ventricle might also be called Dandy-Walker's syndrome in a broad sense.

Diagnosis is established by air study or by operative findings. It is a difficult thing to determaine the pathogensis and all of them are not always malformation. For treatment Torkildsen's operations is effective and other shunting operations are also performed as in hydrocephalus.

\title{
S-D-2. Anomaries of the Cranium, with Special Referrence to the Craniostenosis and Microcephalus
}

\author{
Yutaka HoR \\ 2nd Dept. of Surgery, Nara Medical College
}

